\title{
THE
}

\section{Bose-Einstein Condensation of Interacting Gases in Traps with and without Optical Lattice}

S. Chatterjee

University of Rhode Island

A. E. Meyerovich

University of Rhode Island, sfo101@uri.edu

Follow this and additional works at: https://digitalcommons.uri.edu/phys_facpubs

Terms of Use

All rights reserved under copyright.

\section{Citation/Publisher Attribution}

Meyerovich, A. E., \& Chatter, S. (2009). The American Physical Societyjee. Bose-Einstein condensation of interacting gases in traps with and without optical lattice. Phys. Rev. A, 79(3), 033628, 1-5. doi: 10.1103/ PhysRevA.79.033628

Available at: http://dx.doi.org/10.1103/PhysRevA.79.033628

This Article is brought to you for free and open access by the Physics at DigitalCommons@URI. It has been accepted for inclusion in Physics Faculty Publications by an authorized administrator of DigitalCommons@URI. For more information, please contact digitalcommons-group@uri.edu. 


\title{
Bose-Einstein condensation of interacting gases in traps with and without optical lattice
}

\author{
S. Chatterjee and A. E. Meyerovich \\ Department of Physics, University of Rhode Island, Kingston, Rhode Island 02881-0817, USA
}

(Received 28 October 2008; published 30 March 2009)

\begin{abstract}
We compare effects of particle interaction on Bose-Einstein condensation in inhomogeneous traps with and without optical lattice inside. Interaction pushes normal particles away from the condensate droplet, which is located in the center of the trap, toward the periphery of the trap where the trapping potential is large. In the end, the remaining normal particles are squeezed to a quasi-two-dimensional (2D) shell around the condensate droplet, thus changing the effective dimensionality of the system. In the absence of the optical lattice, the index in the temperature dependence of the condensate density at the later stages of the process is close to 2 with a weak dependence on the number of trapped particles. In the presence of the lattice inside the trap, this simple picture breaks down and the index acquires a strong dependence on the number of particles inside the trap, gradually falling from a three-dimensional to a $2 \mathrm{D}$ value with an increase in the number of particles. This change in index is explained by the lattice-driven spread of the condensate droplet and the localization of the narrow-band particles by the trap potential.
\end{abstract}

DOI: 10.1103/PhysRevA.79.033628

PACS number(s): 67.85.Hj, 03.75.Hh, 05.30.Jp

The spectacular experimental discovery of Bose-Einstein condensation (BEC) made the study of alkali-metal gases in traps the focal point in atomic, low-temperature, and condensed-matter physics. For the first time, it became possible to observe some of the phenomena that have been discussed earlier only within theoretical models (see review in [1]). The phenomena in ultracold alkali-metal gases are incredibly rich and combine features inherent to diverse condensed-matter and low-temperature systems (Ref. [2] and references therein) from "classical" superfluid or superconducting systems [2] to spin-polarized quantum gases [3] to Mott transition in the optical lattice [4].

One of the unavoidable features is the inhomogeneity of the trapping potential. The interplay between the repulsive interaction and the trapping potential complicates BEC. It was clear from the beginning $[5,6]$ that the interaction and the trap have opposite effects on condensation: while the trap tends to concentrate the condensate in a narrow region of space around the particle ground state in the trap, the repulsion is responsible for the widening of this condensate droplet. The analytical description of the combined effects tends to be rather elusive, and our previous experience with condensation in homogeneous systems is not very helpful. The problem becomes even more complex in the presence of the optical lattice inside the trap which adds two different localization processes-Mott transition and localization of narrow-band particles by an inhomogeneous potential.

Below we investigate a situation in which it is possible to get an accurate semianalytical picture of the condensation in trapped interacting gases. The main goal of the paper is comparison of the condensate formations in traps with and without an optical lattice inside. The main attention is paid to the index in the temperature dependence of the condensate fraction and to the size of the condensate droplet. It turns out that this index is not universal even for a low-density gas. What is more, the effective dimensionality of the problem depends on the presence of the optical lattice and changes with condensation, making the later stages of BEC different from initial.

We start from a more conventional and transparent prob- lem of BEC in trapped gases in the absence of the optical lattice, and add the complications associated with the optical lattice later on. A similar calculation without the optical lattice was performed in Ref. [7] though at a noticeably lower population of the trap. This difference strongly affects the results. We assume that the density is still sufficiently low to neglect the interaction before the onset of condensation even in the center of the trap. In computations, this condition limits the total number of particles in our trap, $N$, to $N<10^{6}$. This also means that the critical temperature $T_{c}$ for the onset of condensation is practically unaffected by the interaction. The interaction is brought into play only after the start of condensation, at $T<T_{c}$, since the particles condensate in the center of the trap, making the density in the center large. Thus, the interaction, which is proportional to the particle density, is large only in and around the condensate droplet. The normal particles are pushed out by the dense condensate toward the periphery of the trap where the interaction is negligible. However, the further particles move away from the center, the higher is the gradient of the trapping potential, which is responsible for the force pushing the normal particles back toward the trap center. Therefore, at the later stages of BEC, the majority of remaining normal particles are distributed in an almost two-dimensional (2D) shell around the condensate droplet and the dimensionality of the condensation problem changes from three dimensional (3D) in the beginning of the condensation to quasi-2D later on.

We consider a 3D harmonic trap with a single-particle ground state of frequency $\omega$ and spatial size $\sigma_{0}$ (the axial asymmetry of experimental traps is largely irrelevant in our context). Without interaction, BEC starts at $T_{c}$ $=0.941 \hbar \omega N^{1 / 3}[8]$ and the initial size of the condensate droplet is $\sigma_{0}$. Particle repulsion increases the size of the condensate droplet with $N_{c}(T)$ particles to $\sigma(T)$. Then the potential well for normal particles $U(r)$ has a shell-type structure,

$$
U(r, T)=\frac{1}{2} \hbar \omega\left[\frac{r^{2}}{\sigma_{0}^{2}}+\frac{N_{c} \sigma_{0}^{3}}{N_{0} \sigma^{3}} \exp \left(-\frac{r^{2}}{\sigma^{2}}\right)\right],
$$

where $N_{0}=(\sqrt{\pi} / 8) \omega m \sigma_{0}^{3} / \hbar a_{s}$ and we assume that the condensate density is Gaussian with the variational parameter $\sigma$. 
(In Ref. [7] the shape of the condensate wave function is calculated self-consistently.) The number of normal particles, $N_{n}(T)=N-N_{c}(T)$, is determined from the condition $\mu=0$. The size of the condensate droplet, $\sigma(T)$, can be obtained from minimization of the condensate energy, which includes repulsion, in a way similar to that done in Ref. [5]. There are several reasons that warrant exclusion of the interaction of normal particles between themselves from Eq. (1). First, for less than $10^{5}$ particles in a trap, the interaction of the normal particles is negligible even in the trap center before condensation. Even for larger $N$, the number of the normal particles on the later stages of the condensation is small. Finally, the density of the normal particles is suppressed even more by repulsion from the condensate droplet which spreads them through a large shell around the droplet, $4 \pi \sigma^{2} \sigma_{0}$, instead of concentrating them near the center in the volume $(4 \pi / 3) \sigma_{0}^{3}$. This gives $N$ at least an extra order of magnitude for which we can neglect the interaction of normal particles.

$N_{0}$ in Eq. (1) is the minimal number of particles in the condensate that is sufficient to create a strong repulsive core in the center of the trap. When $N>N_{c} \gg N_{0}$ the normal particles are pushed away from the center by the repulsive core [Eq. (1)] into a potential valley surrounding the condensate droplet. For $\mathrm{Rb}$ in a trap with $\omega=24 \mathrm{~Hz}$, the values $a_{s}$ $=58.2 \AA$ and $\sigma_{0}=2.2 \times 10^{-6} \mathrm{~m}$, and the critical number $N_{0}$ that changes the topology of the normal cloud is $N_{0} \approx 84$. The center of the trap becomes inaccessible for normal particles when $T$ is much smaller than the repulsion from the core. Using $T_{c}$ instead of $T$ and $N$ instead of $N_{c}$, one gets $\sigma^{3} N_{0} / \sigma_{0}^{3} \ll N^{2 / 3}$ and the critical value of $N_{c}$ is around $10^{5}$. All this means that our results are applicable for $N$ in the range $10^{4}-10^{6}$.

We are able to obtain a semianalytical description of the situation (cf. Refs. [6,7]). At the later stages of the condensation, potential (1) forms a distinct valley away from the center of the trap as soon as $N_{c} \gg N_{0}$ and equations for $N_{c}(T)$ and $\sigma(T)$ reduce to

$$
\begin{gathered}
\chi=\sqrt{2}-\frac{\sigma_{0}^{4}}{\sqrt{2} \sigma^{4}}=\frac{4 a_{s} \sigma_{0}^{4}}{\sqrt{\pi} \sigma^{5}} N_{c}, \\
N_{c}=N-\sum_{n=1}\left\{\exp \left[\widetilde{\beta}\left(n+\frac{1}{2}\right) \lambda\right]-1\right\}^{-1} \\
-\sum_{n, l=0} \frac{2 l+1}{\exp \tilde{\beta}\left[\left(n+\frac{1}{2}\right) \lambda+\left(l^{2}+l\right) \frac{\sigma_{0}^{2}}{2 \sigma^{2}} \ln 2 \chi\right]-1},
\end{gathered}
$$

with $\widetilde{\beta}=\hbar \omega / T$ and $\lambda=\sqrt{2 \ln (2 \chi)}$. The summation provides the temperature dependencies $N_{c}(T)$ and $\sigma(T)$.

We found that the condensate fraction at the later stages of condensation can be given as

$$
N_{c} / N=1-\left(T / T_{c}^{*}\right)^{\alpha}
$$

with a relatively high accuracy. The important feature of Eq. (3) is that the temperature is normalized not by the critical temperature $T_{c}$ for the onset of condensation but by a differ-

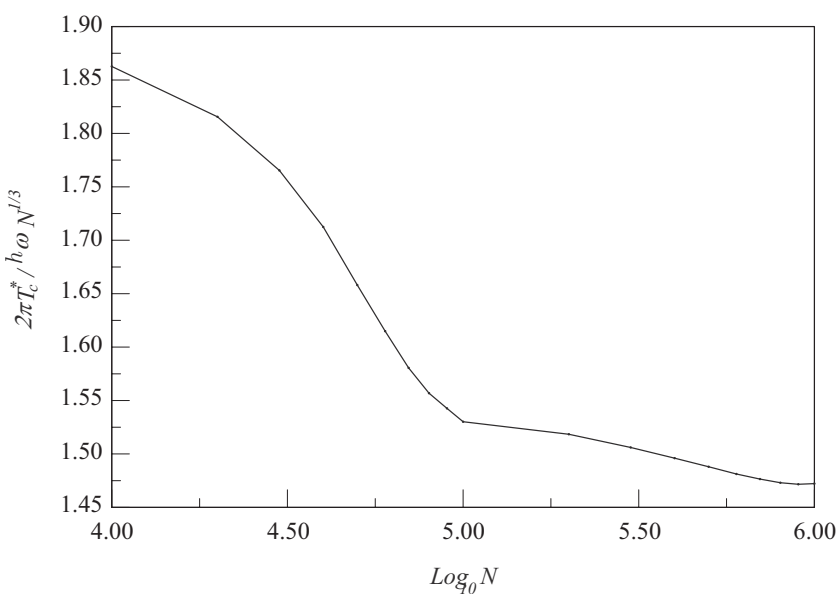

FIG. 1. Density dependence of the reduced critical temperature $T_{c}^{*} / \hbar \omega N^{1 / 3}$ [Eq. (3)]. For a noninteracting gas in a $3 \mathrm{D}$ harmonic trap, this ratio should be 0.91 .

ent value $T_{c}^{*}$. Since the squeezing of the normal particles toward the fringes of the trap accelerates with the number of particles in the condensate $N_{c}$, the normal shell narrows with increasing $N_{c}$ and, therefore, $N$. As a result, the effective temperature $T_{c}^{*}$ should be higher than $T_{c}$ and increase with increasing $N$. Dependence of $T_{c}^{*}$, or more precisely $T_{c}^{*} / \hbar \omega N^{1 / 3}$, on $N$ is presented in Fig. 1. For comparison, the critical temperature $T_{c}$ for noninteracting particles in a $3 \mathrm{D}$ harmonic trap is $T_{c}=0.9 \hbar \omega N^{1 / 3}$ [8]. The authors of [7], who were working with a much smaller number of particles in the trap, $N<10^{4}$, did not observe any difference between $T_{c}$ and $T_{c}^{*}$.

The striking change in behavior of $T_{c}^{*}(N)$ in Fig. 1 occurs at $N$ for which $T_{c} \sim \frac{1}{2} \hbar \omega\left(N_{c} \sigma_{0}^{3} / N_{0} \sigma^{3}\right)$. At higher densities the repulsion from the condensate droplet keeps the normal particles near the bottom of the potential valley around the droplet. At lower densities, the normal particles spread out and can even reach the center of the trap. An anomaly at the same threshold density is also observed in $\alpha(N)$ (Fig. 2), though the index $\alpha$ remains very close to the value of 2 and is practically independent of $N, \alpha=2.02 \pm 1 \%$, in a wide range

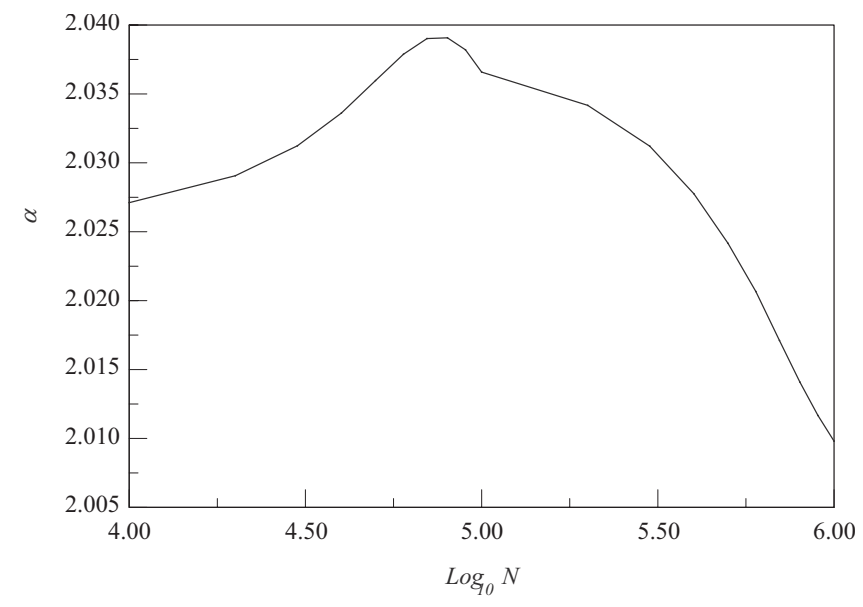

FIG. 2. Density dependence of the index $\alpha$ [Eq. (3)]. For a noninteracting gas in a $3 \mathrm{D}$ harmonic trap, this index should be 3 . 
of $N$ from $10^{4}$ to $10^{6}$. Such a weak dependence $\alpha(N)$ is surprising for a nonlinear problem of this nature. The residual temperature dependence $\alpha(T)$ lies within the same error bars.

For comparison, the same index in Ref. [7] was much higher, around 2.3, which reflects the fact that at much lower occupancy of the trap, as in [7], the dimensionality of the problem is still far away from a $2 \mathrm{D}$ one. Another major difference with Ref. [7] is that we do not see any residual normal fraction, especially in the center of the trap-our condensate density is too high to allow any normal component in the center.

Our results confirm the evolution of the effective dimensionality from 3D, for which $\alpha=3$, to an almost perfect 2D value and the effective narrowing of the trap during condensation.

Using the above results as a reference point, let us turn to BEC in a trap with an optical lattice with a period $a_{0}$ inside. The situation with an optical lattice (Ref. [9] and references therein) inside the trap is much more complex. Here one deals with the Hubbard Hamiltonian, modified by the trap potential, and can encounter two localization effects: the localization of narrow-band particles by an inhomogeneous potential and the Mott transition [10] which requires full occupancy of the lattice sites. The latter can occur with lowering of the temperature when particles gravitate toward the bottom (center) of the trap. With sufficiently strong on-site repulsion, the localization is practically inevitable for the condensate in the center of the trap though, of course, the Mott transition is sensitive to the trap profile $[10,11]$. However, in contrast to the ground-state particles, it is possible to disregard the Mott transition for the normal cloud, surrounding the condensate, due to the increased size of the condensate droplet in comparison to the system without the lattice (see below).

Condensation can be easily understood qualitatively for low initial density of particles $n a_{0}^{3} \ll 1$ and strong on-site repulsion when the condensation starts at the same temperature $T_{c}$ as in the absence of the interaction. The condensate forms in the center of the trap and rapidly expands in size because of the strong on-site repulsion which tends to keep the density $n_{c} a_{0}^{3} \approx 1$. Though the strength of the individual particleparticle repulsion does not depend on the presence of the optical lattice, all the particles in this lattice are located in or around lattice wells and are closer to each other than when they are spread continuously throughout the trap without the lattice inside. As a result, the effect of repulsion in the trap with the lattice is stronger and the size of the condensate droplet, $\sigma \sim a_{0} N_{c}^{1 / 3}$, should be larger than $\sigma_{\max } \sim(2-5) \sigma_{0}$ for traps without the optical lattice (Fig. 3). We will not dwell on potential "freezing" of the condensate resulting from the Mott transition and will concentrate on the condensation of the normal gas outside the condensate droplet.

The main changes in our approach are associated with the band nature of the energy spectrum for particles in the optical lattice and a more complicated form of the wave functions. For the sake of comparison, in numerical computations we use a similar set of parameters: the trap potential and the particle scattering remain the same. For the particle effective mass we use in most of our computations the value $[4,10]$ $m^{*}=16 m$.

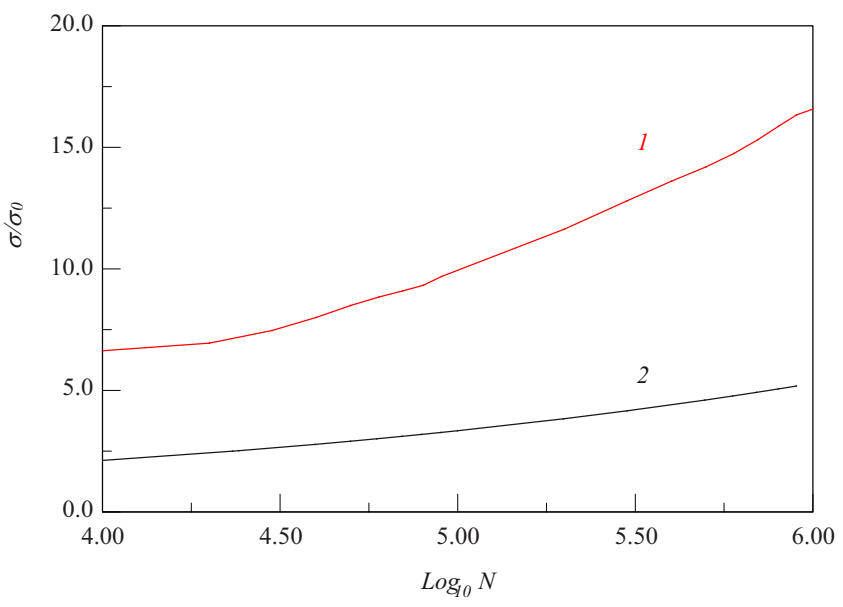

FIG. 3. (Color online) Size of the condensate droplet $\sigma(T=0)$ relative to the size of the trap $\sigma_{0}, \sigma / \sigma_{0}$, with (curve 1) and without (curve 2) the optical lattice as a function of the number of particles in the trap. The scattering lengths and effective masses are identical in both cases. Parameters of the lattice and the trap are given in the text.

The single-particle spectrum in the optical lattice $\epsilon(\mathbf{p})$ has a band structure with a bandwidth $\Delta$. The effect of the trapping potential $U_{\mathrm{tr}}(r)=\frac{1}{2} \hbar \omega\left(r / \sigma_{0}\right)^{2}$ on the particles with narrow bands results in localization of particles with energy $E$ $=\epsilon(\mathbf{p})+U_{\text {tr }}(r)$ in 2D shells $r_{\min } \leq r \leq r_{\max }$ of the thickness $\ell(r) \sim(\Delta / \hbar \omega) \sigma_{0}^{2} / r$ with $r_{\min , \max }$ given by equations $E$ $= \pm \Delta / 2+U_{\operatorname{tr}}\left(r_{\min , \max }\right)$. An exception is the center of the trap, where the gradient of the potential is small. Since such localization suppresses the accessibility range of narrow-band particles, the density in each point contains the contributions from the particles in a finite range of energies that are localized close to this point. For example, since only the particles with very low energies, $E<\Delta$, can reach the center of the trap, the density in the center is suppressed in comparison with the trap without the optical lattice inside.

The particle wave function consists of three regions: rapid oscillations within its classically accessible shell and two attenuating tails beyond the classical turning points. The wave function $\Psi=\Psi_{l m}(E)$ for a particle with the energy $E$ decays relatively slowly beyond the turning point $r_{\max }$ as the Airy function $\mathrm{Ai}$,

$$
\begin{aligned}
\Psi= & B \exp \left(i \pi\left[\frac{E-\Delta / 2-l(l+1) \hbar^{2} / 2 m^{*} d^{2}}{A a}\right]\right) \\
& \times \operatorname{Ai}\left(\tilde{r}-\frac{E-l(l+1) \hbar^{2} / 2 m^{*} d^{2}}{A a}\right) Y_{l m}(\theta, \phi),
\end{aligned}
$$

and similarly near $r_{\min }$. Here $B(E)$ is the normalization coefficient, $m^{*}$ is the effective mass near the band minimum, $A$ $=\hbar \omega r_{\max } / \sigma_{0}^{2}$, the dimensionless coordinate $\tilde{r}=r / a$ has a local length scale $a=\left(\hbar^{2} / 2 m^{*} A\right), Y_{l m}(\theta, \phi)$ are the usual angular harmonics, and $d$ is the position of the minimum of potential (1). The spatial distribution of particles should be calculated taking into account all three regions since for relatively shallow traps the contribution from the tails of the wave function can be large. As a result, the density distribution of particles 


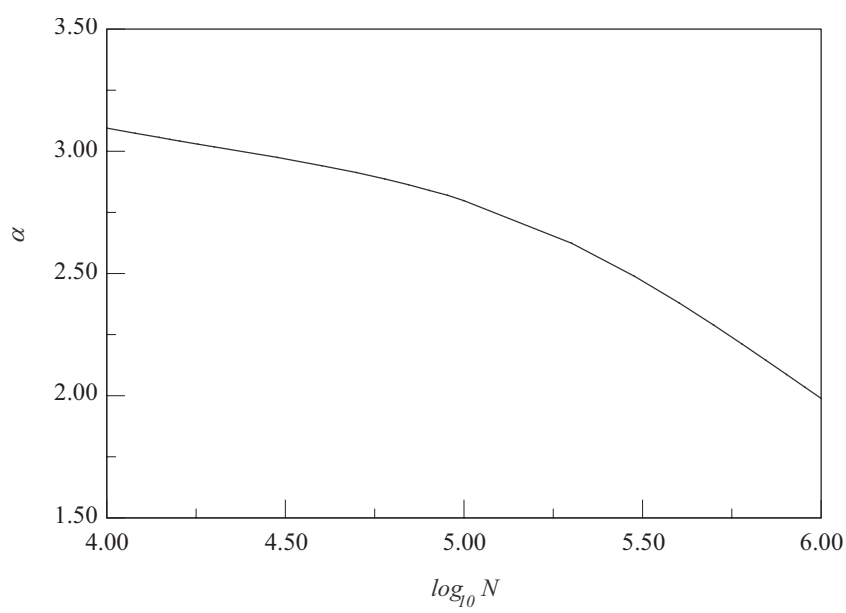

FIG. 4. Index $\alpha$ [Eq. (3)] as a function of the number of the trapped particles in the presence of the optical lattice. Parameters of the lattice used in the computation are given in the text.

inside the trap becomes a much more complicated function of temperature than in the case without the optical lattice.

As above, we start from the situation when the particle density above condensation is low and the (Hubbard) repulsion in the normal phase is negligible. The condition of low density allows us also to disregard the Mott transition in the normal phase [12]. Since the particles in the optical lattice are located mostly on the lattice sites of the size $a_{0}$ rather than spread more or less uniformly, the repulsion is more effective than without the lattice. This means that the size of the condensate droplet, $\sigma(T)$, should be larger than in the absence of the lattice. This is illustrated in Fig. 3, which presents the ratio $\sigma(T=0) / \sigma_{0}$ for identical traps with (curve 1) and without (curve 2) the optical lattice. The scattering amplitude $a_{s}$, which is responsible for repulsion, is the same in both cases.

This seemingly innocuous lattice-driven change in the size of the condensate droplet leads to major changes in the condensation process. Even such a relatively small increase in $\sigma$ can eliminate a repulsive bump [Eq. (1)] in the center of the trap. Indeed, this bump disappears when $\sigma^{5}$ $>\sigma_{0}^{5}\left(N_{c} / N_{0}\right)$. At these values of $\sigma$ the potential (almost) restores its original parabolic structure in the central area despite the presence of the condensate. Since the value of $N$, and therefore $N_{c}$, in our calculations never exceeds $10^{6}$ and $N_{0} \lesssim 100$, the potential remains parabolic for $\sigma / \sigma_{0}>10$. As a result, presence of the optical lattice strongly affects the in$\operatorname{dex} \alpha$ [Eq. (3)], which experiences a much more noticeable change than the condensate droplet size $\sigma$ (Fig. 4).

This density dependence $\alpha(N)$ is dramatically different from the one in Fig. 2 for the trap without the optical lattice inside.

In Fig. 4, $\alpha$ starts from a 3D value at small density of particles, which is understandable since there is no repulsive core in the center. With increasing number of particles the

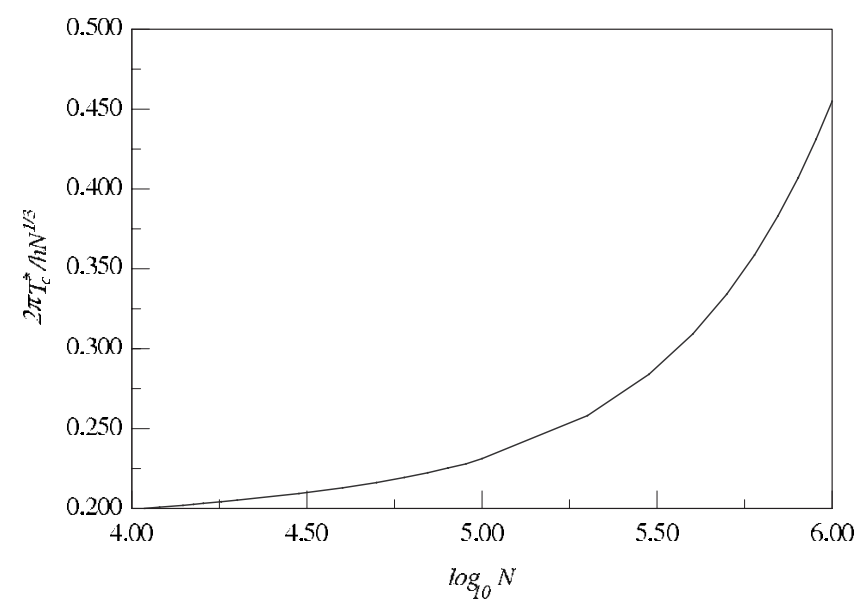

FIG. 5. Density dependence of the reduced critical temperature $T_{c}^{*} / \hbar \omega N^{1 / 3}$ [Eq. (3)] in the presence of the optical lattice. The behavior of $T_{c}^{*}$ is drastically different from a similar trap without the optical lattice (Fig. 2).

size of the condensate droplet grows, leaving fewer normal particles in the central area and gradually reducing $\alpha$ to its quasi-2D value. What is not clear is why does $\alpha$ continue to decline with a further increase in $N$. However, since our approach loses accuracy beyond $N \sim 10^{6}$, we do not present these data in the figure. Note, that if one also plots in Fig. 4 the curve $\alpha(N)$ for a system without the optical lattice from Fig. 2, all its residual dependence on $N$ becomes invisible in the scale of Fig. 4. This difference in dependences of the index $\alpha$ on the number of trapped particles between Figs. 4 and 2 is due mostly to the major qualitative changes in the wave functions imposed by the lattice symmetry. Computations with a smaller effective mass did not lead to any major changes in behavior of $\alpha(N)$.

In general, the decrease in $\alpha(N)$ is accompanied by an increase in $T_{c}^{*}(N)$, which in the presence of the optical lattice grows much faster than $N^{1 / 3}$ dependence inherent to a free gas in a trap (Fig. 5; cf. Fig. 1).

In summary, we calculated the index for a temperature dependence of the condensate fraction for interacting gas inside harmonic trap. The results for traps without the optical lattice inside are quite clear: the repulsion from the condensate droplet pushes normal particles away from the center of the trap and concentrates them in a relatively thin shell around this droplet. Then the condensation becomes almost quasi-2D with the index $\alpha \approx 2$. The presence of the optical lattice inside the trap changes the situation in a major way. The index $\alpha$ acquires a strong dependence on the number of particles inside the trap and gradually falls from a 3D to a 2D value with an increase in the number of particles. This change in the index, which is caused by the presence of the optical lattice, is explained by the wider spread of the condensate droplet and the localization of the narrow-band particles by the trap potential. 
[1] E. A. Cornell and C. E. Wieman, Rev. Mod. Phys. 74, 875 (2002).

[2] A. J. Leggett, Rev. Mod. Phys. 73, 307 (2001); F. Dalfovo, S. Giorgini, L. P. Pitaevskii, and S. Stringari, ibid. 71, 463 (1999); I. Bloch, Nature (London) 453, 1016 (2008).

[3] H. J. Lewandowski, D. M. Harber, D. L. Whitaker, and E. A. Cornell, Phys. Rev. Lett. 88, 070403 (2002); M. Ö. Oktel and L. S. Levitov, ibid. 88, 230403 (2002); J. N. Fuchs, D. M. Gangardt, and F. Laloë, ibid. 88, 230404 (2002); J. E. Williams, T. Nikuni, and C. W. Clark, ibid. 88, 230405 (2002); A. Kuklov and A. E. Meyerovich, Phys. Rev. A 66, 023607 (2002); T. K. Koponen, T. Paananen, J.-P. Martikainen, and P. Törmä, Phys. Rev. Lett. 99, 120403 (2007).

[4] M. Greiner, O. Mandel, T. W. Hansch, and I. Bloch, Nature (London) 415, 39 (2002); V. A. Kashurnikov, N. V. Prokof'ev, and B. V. Svistunov, Phys. Rev. A 66, 031601(R) (2002); A. Kuklov, N. Prokof'ev, and B. Svistunov, Phys. Rev. Lett. 92, 050402 (2004).

[5] G. Baym and C. J. Pethick, Phys. Rev. Lett. 76, 6 (1996).

[6] S. Giorgini, L. P. Pitaevskii, and S. Stringari, Phys. Rev. A 54, R4633 (1996).

[7] D. A. W. Hutchinson, E. Zaremba, and A. Griffin, Phys. Rev. Lett. 78, 1842 (1997).
[8] V. V. Goldman, I. F. Silvera, and A. J. Leggett, Phys. Rev. B 24, 2870 (1981).

[9] G. Raithel, G. Birkl, A. Kastberg, W. D. Phillips, and S. L. Rolston, Phys. Rev. Lett. 78, 630 (1997); T. Müller-Seydlitz, M. Hartl, B. Brezger, H. Hänsel, C. Keller, A. Schnetz, R. J. C. Spreeuw, T. Pfau, and J. Mlynek, ibid. 78, 1038 (1997); S. E. Hamann, D. L. Haycock, G. Klose, P. H. Pax, I. H. Deutsch, and P. S. Jessen, ibid. 80, 4149 (1998); L. Guidoni, C. Triche, P. Verkerk, and G. Grynberg, ibid. 79, 3363 (1997); K. I. Petsas, A. B. Coates, and G. Grynberg, Phys. Rev. A 50, 5173 (1994); I. H. Deutsch and P. S. Jessen, ibid. 57, 1972 (1998).

[10] D. Jaksch, C. Bruder, J. I. Cirac, C. W. Gardiner, and P. Zoller, Phys. Rev. Lett. 81, 3108 (1998).

[11] G. K. Campbell, J. Mun, M. Boyd, P. Medley, A. E. Leanhardt, L. G. Marcassa, D. E. Pritchard, and W. Ketterle, Science 313, 649 (2006); G. G. Batrouni, V. Rousseau, R. T. Scalettar, M. Rigol, A. Muramatsu, P. J. H. Denteneer, and M. Troyer, Phys. Rev. Lett. 89, 117203 (2002); B. DeMarco, C. Lannert, S. Vishveshwara, and T. C. Wei, Phys. Rev. A 71, 063601 (2005); P. Sengupta, A. Raghavan, and S. Haas, New J. Phys. 9, 103 (2007).

[12] A. E. Meyerovich, Phys. Rev. A 68, 051602(R) (2003). 